that work may have on the transition between one's work and non-work domains (i.e., commuting). We sought to examine the impact of work-related rumination (WRR) as a cognitive distracting mechanism on employee's commuting safety behaviours (CSBs) and the potential buffering effects of personal-protective factors (commute-as-relaxation) as employee's commute from work to home.

Methods Data were collected via a baseline and 10 day daily diary study (two weeks, Monday - Friday) from employees $(n=106)$ who worked full-time and commuted via private vehicle on a daily basis between March - April 2015. Data were analysed using Mplus version 7.3.1. and results were limited to the baseline and first week of daily diary collection. Result On average, employees experience a decrease in risky CSBs over the work week, with a rise in risky CSB occurring from Thursday to Friday. Travel-speed disruptions and WRR significantly impacted the trajectory of risky CSBs, such that travel-speed disruptions and engaging in WRR resulted in riskier CSBs. Additionally, employees who view the commute as time for relaxation engaged in less risky CSBs than their counterparts. The final model fit the data well $\left[\chi^{2}(53)=67.81\right.$, $\mathrm{p}=0.08$, RMSEA $=0.058, \mathrm{CFI}=0.94, \mathrm{TLI}=0.93, \mathrm{SRMR}=0.07]$. Discussion Results indicated that employees who engage in WRR engage in riskier CSBs over the course of the working week. However, employees who view their commutes as time for relaxation engage in less risky CSBs over their counterparts. The findings of this study indicate the need for workplace intervention techniques that target the negative spill-over of WRR into employees' commute time, as well as highlights the need for work-recovery mechanisms.

\section{QUALITY OF WORK LIFE AMONG DOCTORS IN THE UNIVERSITY HOSPITALS IN HEALTH AREA 7 OF THAILAND}

N Chaiear*, W Soonthornvinit, S Boonjaraspinyo. Division of Occupational Medicine, Department of Community Medicine, Faculty of Medicine, Khon Kaen University, Khon Kaen, Thailand

\subsection{6/oemed-2018-ICOHabstracts. 1753}

Introduction Despite extensive information regarding quality of work life in medical residents in Thailand, however no study has examined the quality of work life (QoWL) amongst hospital doctors in Thailand. The aim of study is to evaluate the QoWL of hospital doctors in the university hospitals in the health area 7.

Methods A descriptive study was conducted among all 910 doctors in the university hospitals in health area 7 of from May to June 2017. The data was collected by web-based Thai Work-Related Quality of Life Scale - 2 (Thai WRQLS-2).

Results Response rate was 39.1\% (356/910). The mean rating of qowl was 106.36 out of 155 (SD 16.09). 61.0\% of doctors rated their QoWL as moderate scale. The eight sub-factors were rated as moderate to high for control at work, homework interface, and working conditions; moderate and high for general well-being; high down to moderate for employee engagement, job and career satisfaction, and overall QoWL; and moderate down to low for stress at work. Details of factors which might influence their QoWL is being analysed and will be updated soon.

Conclusion This is the first study that assessed the QoWL among doctors in the university hospitals in health area 7.
Majority of doctors rated QoWL as moderate. The sub-factor which rated to be the lowest score was stress at work.

\section{HOLISTIC APPROACH TOWARDS EMPLOYEES' LIFESTYLE MANAGEMENT THROUGH WELLNESS PROGRAMS TO REDUCE NON COMMUNICABLE DISEASES BURDEN}

Divyesh Chauhan*. Centre for Innovation and Technology, Hero MotCorp Limited, Jaipur, India

\subsection{6/oemed-2018-ICOHabstracts. 1754}

Introduction With the increased levels of competition in the business, employees today are being faced with a serious threat of non-communicable diseases like Diabetes, Blood Pressure, Heart diseases etc. Various factors affecting the lifestyle are Bad Food Habits, Smoking, stress, overweight, sedentary lifestyle etc. To halt the progression of unhealthy lifestyle and its outcome, a tailored education and lifestyle management interventions is designed which directly or indirectly lower the risks and improve outcomes.

Methods All the employees who undergo for annual health examination are considered in the study. From annual health examination data the age, past history, family history smoking and alcohol habits, BMI, Blood pressure, Fasting Blood sugar, and Cholesterol and exercises habit were considered. Accordingly the employees were divided in mild, moderate and severe risk group.

A tailored and personalised intervention programs were conducted with targeting modifiable risk factors and behaviour changes focus on encouraging physical activity, weight management, smoking cessation, and stress management. Multi modal communication like awareness talks, Diet Counselling, Health tips, Health talks, guest lectures, structured health programs, Yoga session, one to one counselling session electronic media, movie etc. helped in raising awareness and changing attitude towards lifestyle management.

Results The health status of employees has shown tremendous improvements month on month basis as they have become more sensitised and aware towards their health issues and its consequences. Improved work culture, reduction in sickness absenteeism, employees coming forward for counselling sessions.

Conclusion Poor Lifestyle management is one of the main culprits in rising numbers of non-Communicable disease. Holistic approach is required to improve the unhealthy lifestyle and to change the attitude toward it. Successful Wellness programs are one of the most effective tools for the same.

\section{SOCIAL DETERMINANTS AND GENDER DIFFERENCES IN WORK-LIFE CONFLICT: THE EFFECTS OF AGE AND MARRIAGE}

${ }^{1}$ Ceyda Sahan, ${ }^{1,2}$ Yucel Demiral. 'Occupational Medicine Department, Dokuz Eylul University, Izmir, Turkey; ${ }^{2}$ Public Health Department, Dokuz Eylul University, Izmir, Turkey

\subsection{6/oemed-2018-ICOHabstracts.1755}

Introduction Work-life conflict (WLC) is defined as '...a form of inter-role conflict in which work and family demands are mutually incompatible, meeting demands of both the domains is difficult' and has become a new topic of research interest. 
The aim of the study was evaluating social determinants and gender differences in WLC.

Methods The study has been carried out in three workplaces (Call centre, plastic and metal industry) in 807 workers between 01.08.2016 and 01.01.2017. Independent variables were age, gender, maritual status, educational status and occupational class. Dependent variable was WLC. Copenhagen Psychosocial Questionnaire-3 were used to evaluate work life conflict. We analysed the association between social determinants and work life conflict by using chi-square test and logistic regression.

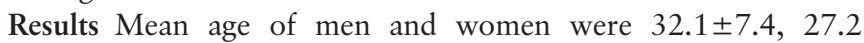
\pm 6.6 , respectively. $43.0 \%$ were women. WLC was found higher in women $(55.9 \%)$ than men $(42.4 \%) \quad(\mathrm{p}<0.001)$. White collar, younger and high educated workers had higher risk of having WLC than their reference groups $(p<0.05$ for all comparisons). Logistic regression analysis revealed that, being younger and married were independently associated with WLC $(\mathrm{p}<0.05)$ in women. Being younger was independently associated with WLC $(p<0.05)$ in men. There were no association between educational status or occupational class and WLC in both gender.

Conclusion In this study, an inverse relationship was found between age of the employees and WLC. Marital status is important factor in women but not men in terms of WLC.

\section{INFLUENCE OF FACTORS OF WORK-LIFE BALANCE ON WORK ENGAGEMENT AMONG JAPANESE WORKERS}

${ }^{1} \mathrm{~K}$ Hayashi ${ }^{*},{ }^{2} \mathrm{~T}$ Nagata. ${ }^{1}$ Ewel, Inc., Japan; ${ }^{2}$ Institute of Industrial Ecological Sciences, University of Occupational and Environmental Health, Japan

\subsection{6/oemed-2018-ICOHabstracts.1756}

Introduction Studies on work engagement (WE) tend to be related to work factors. Few studies exist on non-work factors such as work-life balance(WLB), living condition in family, and leisure time. Currently, Japan has experienced changes in demographic factors due to the declining birthrate and ageing population and in psychological factors due to diversification of sense of values. Moreover, as the time outside of working hours increases due to national policies on working style and working time management reform, intervention for non-work factors, especially factors of WLB, will become important for improving WE in the future.

Methods Data on 10060 persons among Japanese workers, collected using an employee questionnaire administered at the Collaboration Health Study Group (11 companies; 29194 persons), were analysed after excluding missing data. First, the Spearman's correlation coefficient was calculated to examine the relationship between WE and seven items related to factors of WLB (number of acquired paid vacation days, number of acquired paid vacation days by purpose, leisure time [estimated from overtime hours], degree of satisfaction of time allocation, recovery experiences, buffer factors other than the workplace, and sleeping hours). Items with high correlation coefficients were subjected to a single regression analysis.

Results Of the factors of WLB, a weak correlation was noted for degree of satisfaction with time allocation $(r=0.366)$. Moreover, a single regression analysis revealed the items in the order of degree of satisfaction with time allocation $(\beta=0.149)$.

Conclusion Among the factors of WLB, higher satisfaction with time allocation and subjective sense of health corresponded to higher WE. In future, we plan to continue to examine how to use time and leisure content, and to use the findings to develop concrete measures for occupational health and human resources management.

\section{THE ASSOCIATION BETWEEN WORK-FAMILY SPILLOVER AND QUALITY OF SLEEP: A PROSPECTIVE STUDY OF JAPANESE WORKERS}

${ }^{1}$ Yui Hidaka*, ${ }^{2}$ Akihito Shimazu, ${ }^{3}$ Kotaro Imamura, ${ }^{4}$ Noboru Iwata, ${ }^{5}$ Kyoko Shimada, ${ }^{6}$ Masaya Takahashi, ${ }^{7}$ Masahito Tokita, ${ }^{8}$ Izumi Watai, ${ }^{3}$ Kazuhiro Watanabe, ${ }^{3}$ Norito Kawakami. 'Department of Psychiatric Nursing, Graduate School of Medicine, The University of Tokyo, Tokyo, Japan; ${ }^{2}$ Center for Human Social Science, Kitasato University College of Liberal Arts and Science, Sagamihara, Japan; ${ }^{3}$ Department of Mental Health, Graduate School of Medicine, The University of Tokyo, Tokyo, Japan; ${ }^{4}$ Department of Psychology, Hiroshima International University, Hiroshima, Japan; ${ }^{5}$ Department of Social Psychology, Faculty of Sociology, Toyo University, Tokyo, Japan; ${ }^{6}$ National Institute of Occupational Safety and Health, Kawasaki, Japan; ${ }^{7}$ Department of Psychiatric and Mental Health Nursing, Graduate School of Nursing, Chiba University, Chiba, Japan; ${ }^{8}$ Department of Nursing, Graduate School of Medicine, Nagoya University, Nagoya, Japan

\subsection{6/oemed-2018-ICOHabstracts. 1757}

Introduction Quality of Sleep has been linked to poor health. While work-family spillover is associated with health problems and impaired work performance of workers, previous findings were inconsistent on, the association between work-family spillover and sleep quality. No study was conducted in nonwestern countries. The objective of this study was to investigate the prospective association between work-family spillover and sleep quality among Japanese workers.

Methods The database used in the study was from a 2 year prospective cohort study. In the baseline survey (T1) in 2011, questionnaires were sent to 1356 workers living in two wards of Tokyo, and 753 (56\%) responded, and 489 completed all scales and items used the study. A follow-up survey was conducted in 2013, with 224 (46\%) respondents out of the 489 completers at T1; 176 completed all scales. The questionnaire (both T1 and T2) included self-reported instruments of workfamily spillover (the Survey Work-home Interaction-NijmeGen, SWING), sleep quality (Pittsburgh Sleep Quality Index, PSQI), and job stressors (Brief Job Stress Questionnaire, BJSQ), and demographic variables (age, sex, education, marital status, and work styles). Multiple linear regression analysis was employed of PSQI scores on SWING scale scores, adjusting for demographic variables and PSQI score at T1. (SPSS version 22).

Results Among the 176 completers, 36\% were men; the average age was 39 years old. Work to family negative spillover (beta $=0.20)$ significantly and positively correlated with sleep quality, after adjusting for the demographic variables $(p=0.020)$. No significant association was observed between the other type of spillover (i.e. work to family positive, family to work positive or negative) and sleep quality. ( $p>0.05)$.

Conclusion This prospective study confirmed the association between work-family negative spillover and sleep quality in a sample of Japanese workers. Work to family negative spillover could be considered a target condition to improve sleep quality of workers. 